\title{
Pulmonary actinomycosis in a male patient with a tracheal bronchus
}

\author{
Cecilia T Costiniuk MD ${ }^{1}$, Nha Voduc MD FRCPC ${ }^{1}$, Carolina de Souza MD PhD²
}

CT Costiniuk, N Voduc, C de Souza. Pulmonary actinomycosis in a male patient with a tracheal bronchus. Can Respir J 2011;18(2): 84-86.

BACKGROUND: Tracheal bronchus is a congenital malformation comprising an abnormal bronchus originating from the trachea or other bronchus. This malformation has been associated with recurrent pneumonia in children, but is rarely associated with infection in adults. Actinomyces species are rare causes of necrotizing pneumonias that often masquerade as malignancy, lung abscesses and tuberculosis.

METHODS AND RESULTS: A case involving a 46-year-old man with a tracheal bronchus and chronic pneumonia syndrome is presented. Bronchialveolar lavage and transthoracic needle biopsy demonstrated the presence of Actinomyces meyeri and Fusobacterium species.

CONCLUSIONS: The present article reports the first documented case of actinomycosis occurring in a patient with a tracheal bronchus.

Key Words: Accessory tracheal bronchus; Actinomyces; A meyeri; Bronchus suis; Congenital lung abnormality; Pulmonary actinomycosis

$\mathrm{T}$ racheal bronchus is a congenital condition characterized by an abnormal bronchus originating from the trachea or right main bronchus and directed to the right upper lobe $(1,2)$. In some cases, the bronchus arises from the right side of the trachea, proximal to the carina, and is referred to as 'bronchus suis' (pig bronchus) because a tracheal bronchus is normally present in swine (1). In children, this condition has been associated with recurrent pneumonia. In intubated patients, this condition has been associated with atelectasis and postobstructive pneumonia due to obstruction of the tracheal bronchus by the endotracheal tube (1). Pulmonary actinomycosis is an anaerobic infection secondary to Actinomyces organisms, which are normal commensals of oral and intestinal flora. Actinomyces species are a rare cause of necrotizing pneumonia, often initially misdiagnosed as malignancy, lung abscesses or tuberculosis $(3,4)$. We present a case involving a 46-year-old man with a tracheal bronchus (bronchus suis) infected with Actinomyces meyeri. To our knowledge, the present report documents the first reported case of pulmonary actinomycosis in a patient with this congenital abnormality. The pathophysiology, diagnosis and management of pulmonary actinomycosis are reviewed.

\section{CASE PRESENTATION}

A 46-year-old man was admitted to hospital for evaluation of a fivemonth history of progressive right-sided pleuritic chest pain and productive cough with purulent blood-tinged sputum. He also reported recent night sweats. Medical history included coronary artery disease, hypertension, type 2 diabetes mellitus, alcohol abuse and 30 pack-year smoking. He had no history of tuberculosis, and previous tests for HIV were negative. He was afebrile with a respiratory rate of 24 breaths/min and an oxygen saturation of $99 \%$ on room air. Crackles were present in the right upper lobe, and his dentition was poor.

Over the five months preceding his admission, the patient presented to the emergency room on two occasions. At the time of the first presentation, he was diagnosed with community-acquired pneumonia and subsequently prescribed clarithromycin, resulting in an equivocal

\section{Une actinomycose pulmonaire chez un homme} ayant une bronche trachéale

HISTORIQUE : La bronche trachéale est une malformation congénitale formée par une bronche anormale provenant de la trachée ou d'une autre bronche. Cette malformation s'associe à une pneumonie récurrente chez les enfants, mais rarement à des infections chez les adultes. Les espèces d'Actinomyces sont de rares causes de pneumonies nécrosantes qui imitent souvent des tumeurs malignes, des abcès pulmonaires et la tuberculose. MÉTHODOLOGIE ET RÉSULTATS : Les auteurs présentent le cas d'un homme de 46 ans ayant une bronche trachéale et un syndrome de pneumonie chronique. Le lavage bronchoalvéolaire et la biopsie transthoracique à l'aiguille ont démontré la présence d'espèces d'Actinomyces meyeri et de Fusobacterium.

CONCLUSIONS : Le présent article rend compte du premier cas documenté d'actinomycose se déclarant chez un homme ayant une bronche trachéale.

response. Three months later, he returned to the emergency room and was prescribed 21 days of amoxicillin/clavulinic acid. With this second course of antibiotics, he reported a notable improvement in his chest pain and sputum production. Unfortunately, his improvement was transient; his symptoms returned shortly after his antibiotic regimen was completed.

During this admission, the patient was initially treated with intravenous levofloxacin for presumptive pneumonia. Chest $\mathrm{x}$-rays demonstrated progressive focal airspace disease with cavitation in the right upper lobe (Figure 1). This was confirmed on contrast-enhanced computed tomography (CT), which also demonstrated ground-glass opacities in the adjacent lung with interlobular septal and bronchial wall thickening. Multiple enlarged mediastinal lymph nodes were present. There was no evidence of pleural disease or chest wall invasion.

Bronchoscopy revealed a small accessory bronchial orifice on the right lateral tracheal wall just proximal to the bifurcation of the trachea into the left and right bronchi. Copious purulent secretions were removed from this tracheal bronchus and bronchoalveolar lavage was performed. The bronchus was carefully inspected to confirm the presence of bronchial mucosa.

Following the bronchoscopy, coronal reformations of previous CT images (Figure 2) confirmed the presence of a tubular air-containing structure arising from the lateral wall of the lower trachea and extending to the right upper lobe lesion, consistent with a tracheal bronchus.

Cytological examination of transthoracic needle aspirate and bronchoalveolar lavage samples did not show any evidence of malignancy. Stains for microorganisms demonstrated filamentous bacteria. Subsequent culture of both aspirate and lavage samples demonstrated the presence of Actinomyces meyeri and Fusobacterium species. Stains for acid-fast bacilli and cultures for Mycobacterium tuberculosis were negative.

When the culture results became available, the patient's antibiotic regimen was changed from levofloxacin to penicillin G 5 million units

${ }^{1}$ Department of Medicine; ${ }^{2}$ Department of Diagnostic Imaging, University of Ottawa, Ottawa, Ontario

Correspondence: Dr Nha Voduc, Division of Respirology, The Ottawa Hospital - General Campus, 501 Smyth Road,

PO Box 211, Ottawa, Ontario K1N 8L6. Telephone 613-737-8198, fax 613-737-8537, e-mail nvoduc@ottawahospital.on.ca 


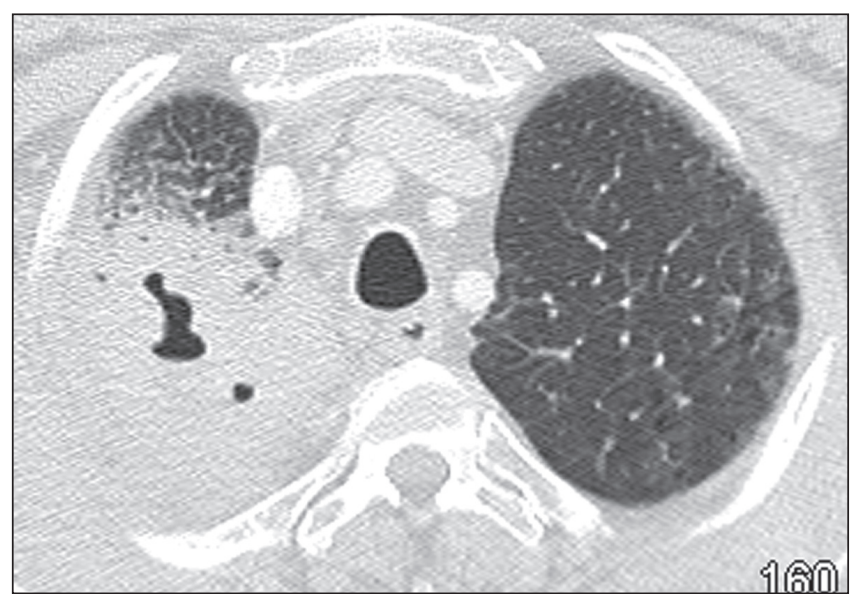

Figure 1) Computed tomography scan of the chest with lung window settings confirming the presence of a cavitary lesion in the right upper lobe. There are surrounding ground-glass opacities and a few septal lines in the periphery of the lung

intravenously every $6 \mathrm{~h}$ and metronidazole $500 \mathrm{mg}$ orally every $8 \mathrm{~h}$; the latter was used to ensure broader anaerobic coverage given the extensively necrotizing nature of the infection. Within $72 \mathrm{~h}$ of starting penicillin and metronidazole, there was clear improvement in sputum production and night sweats. A repeat CT scan, performed after one week of penicillin and metronidazole therapy, demonstrated significant improvement in the areas of consolidation and necrosis. The patient was discharged home on intravenous penicillin and oral metronidazole.

After a total of four weeks of intravenous penicillin and oral metronidazole, the patient returned for outpatient follow-up, reporting a near-complete resolution of sputum production and night sweats. At this point, the patient was switched to penicillin V $500 \mathrm{mg}$ orally every $6 \mathrm{~h}$ and asked to continue on oral metronidazole at $500 \mathrm{mg}$ orally every $8 \mathrm{~h}$. A follow-up bronchoscopy was performed to confirm the presence of a tracheal bronchus and rule out the possibility of a fistula (a possible complication of Actinomyces infection). The bronchoscopy demonstrated a small patent tracheal bronchus lined by normal bronchial mucosa. A third CT of the chest demonstrated further resolution of the consolidation, with some areas of scarring. There was no evidence of pleural disease or chest wall invasion. At this point, metronidazole was discontinued but the patient was maintained on oral penicillin.

The patient was clinically assessed for the final time 24 weeks after initiation of penicillin. By then, he had received four weeks of intravenous penicillin followed by 20 weeks of oral penicillin, for a total duration of penicillin therapy of 24 weeks. The total duration of metronidazole therapy was six weeks. At this point, the patient was essentially asymptomatic, with a normal respiratory examination. A final chest $\mathrm{x}$-ray demonstrated some persisting right upper lobe opacity, compatible with scarring.

\section{DISCUSSION}

Actinomyces organisms are filamentous Gram-positive microaerophilic bacteria that are normal commensals of the oropharynx and gastrointestinal tract. They typically reside in carious teeth, dental plaque, and gingival and tonsillar crypts (3-7). The cervicofacial area is most commonly affected, accounting for $50 \%$ to $60 \%$ of cases (4). The classic description is that of a middle-age man presenting with a large jaw mass (3). Pulmonary actinomycosis is the next most frequent infection, accounting for $15 \%$ to $20 \%$ of reported cases. The remaining infection sites include abdominopelvic (20\%), central nervous system (2\%) and, very rarely, cutaneous, ophthalmic, cardiac, genitourinary and disseminated disease $(3,4)$.

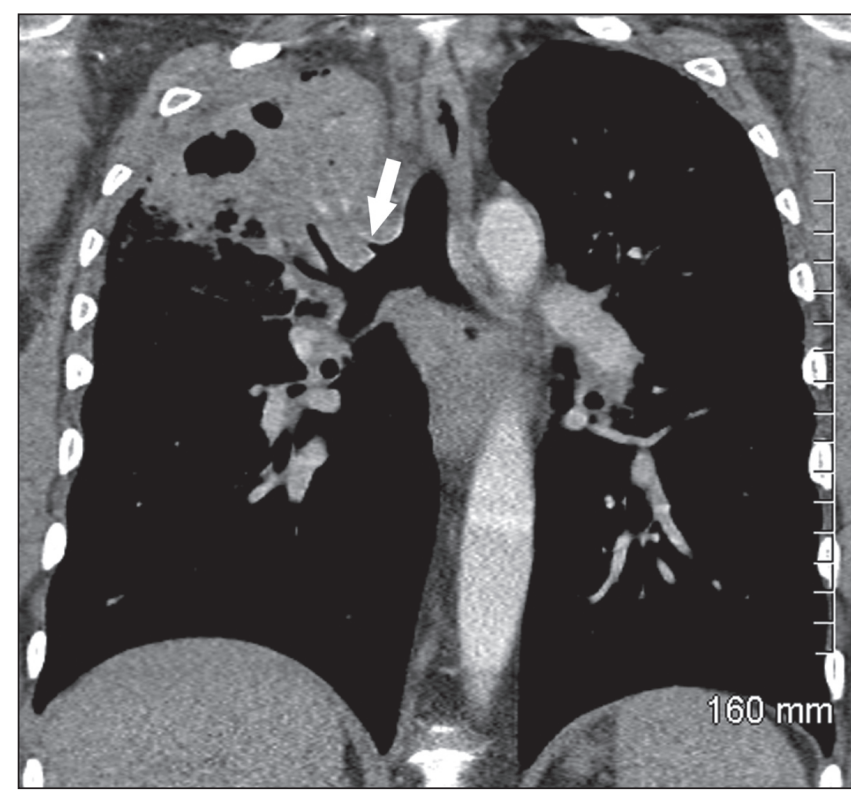

Figure 2) Coronal computed tomography reformation shows a tubular aircontaining structure arising from the lateral wall of the distal trachea (arrow), immediately above the origin of the right upper lobe bronchus and extending to the right upper lobe lesion. Areas of inhomogeneous attenuation, a few opacified vessels and cavitation are seen within the right upper lobe lesion

Among the Actinomyces species, A meyeri has a greater tendency for hematogenous dissemination and lung infection. Infection is believed to result from aspiration of oral and gastric contents, which is supported by the higher prevalence of alcoholism and poor dentition in infected patients, and the bibasilar predominance of the disease radiographically (7-9). Neglecting anatomical boundaries and crossing interlobar fissures, A meyeri induces necrosis, fibrosis and cavitation $(3,5)$. It may further invade the pleura, chest wall, soft tissues and bony structures, and produce sinus tracts (3). Other organisms, such as Fusobacterium species and Bacteroides species, are frequently found in association with the Actinomyces organisms and are believed to enhance its pathogenicity by creating an anaerobic environment in which phagocytosis is inhibited (3). Dyspnea, productive cough and pleuritic chest pain are the typical presenting symptoms $(6,8)$.

Diagnosis is challenging because microbiological identification may be difficult. Actinomyces shares many clinical and imaging findings with chronic suppurative lung infections, and may be mistaken for tuberculosis, nocardiosis, histoplasmosis, blastomycosis or mixed anaerobic infections, in addition to noninfectious etiologies such as pulmonary infarction and malignancy (3-7). Plain x-rays are nonspecific, with a nonsegmental pneumonia usually in the right upper lobe, peripherally crossing fissures. CT of the chest typically demonstrates segmental airspace consolidation with adjacent pleural thickening and microabscesses or necrotic tissue. Invasion of the chest wall may occur and mimic malignancy. The presence of air bronchograms is suggestive of actinomycosis as opposed to malignancy, which often obliterates the bronchi $(3,7)$. Up to one-third of cases of actinomycosis are initially misdiagnosed as malignancy $(3,10)$.

Culture of bronchoalveolar lavage secretions alone is not reliable because positive results may represent contamination from oral flora. Culture samples must be obtained anaerobically with a protected specimen brush. Prolonged incubation for up to 25 days may be required (9). Often, a diagnosis of actinomycosis is made only after resection $(3,4,7,8)$. In many series, six months was the average duration of illness before a definitive diagnosis was made (3). 
The pathophysiology of our patient's disease was probably multifactorial. He had a number of classic risk factors for actinomycosis including alcohol use, tobacco smoking and poor dentition, which predisposed him to infection. It is unknown whether the presence of a tracheal bronchus was also a risk factor for Actinomyces infection in our patient, although it is notable that the infection involved the area of lung served by the tracheal bronchus. At the very least, the tracheal bronchus complicated assessment of this patient's problem by raising the possibility of fistula, a well-described complication of actinomycosis. The second bronchoscopy was performed after four weeks of therapy to verify the presence of the tracheal bronchus and rule out a fistula. To our knowledge, actinomycosis in an adult with a tracheal bronchus has not been previously described.

Our patient's indolent course over several months is typical of pulmonary actinomycosis. Standard treatment of deep-seated actinomycoses consists of intravenous penicillin $G 150,000$ units $/ \mathrm{kg} /$ day to $200,000 \mathrm{U} / \mathrm{kg} / \mathrm{day}$, or $10 \mathrm{million}$ units/day to 20 million units/day, divided three times daily or four times daily, for four to six weeks. This is followed by oral penicillin V $2 \mathrm{~g}$ /day to $4 \mathrm{~g}$ /day, divided three or four times daily, for an additional six to 12 months (11). Metronidazole is frequently added to cover accompanying anaerobic oral flora such as Fusobacterium (11). In some cases, the duration of antimicrobials may be further prolonged if the infection is extensive and involves poorly vascularized or devitalized tissue (5). Surgery may be required for empyemas with discharging fistulae, and rarely to control life-threatening hemoptysis (8). Untreated, it may ultimately be fatal $(5,6)$. However, prognosis is excellent, with cure rates of $90 \%$ with early diagnosis and appropriate antimicrobials $(3,4,6,12)$.

DISCLOSURE: The authors received no funding and have no conflicts of interest to declare for this case report. Ethics approval was not required.

\section{REFERENCES}

1. Doolittle AM, Mair EA. Tracheal bronchus: Classification, endoscopic analysis and airway management. Otolaryngol Head Neck Surg 2002;126:240-3.

2. Ghaye B, Szapiro D, Franchamps JM, Dondelinger RF. Congenital bronchial abnormalities revisited. Radiographics 2001;21;105-19.

3. Mabeza GF, Macfarlane J. Pulmonary actinomycosis. Eur Respir J 2003;21:545-51.

4. Yidiz O, Doganay M. Actinomycosis and nocardia pulmonary infections. Curr Opin Pulm Med 2006;12:228-34.

5. Zijlstra EE, Swart GR, Godfroy FJM, et al. Pericarditis, pneumonia and brain abscess due to combined Actinomyces-Actinobacillus Actinomycetemcomitans infection. J Infect 1992;25:83-7.

6. Fahim A, Teoh R, Kastelik J, et al. Case series of thoracic actinomycosis presenting as a diagnostic challenge. Respir Med CME 2009;2:47-50.

7. Ali HA, Lippman M, Mundathaje U, et al. A young male with history of alcohol abuse with opacified left hemithorax and massive hemoptysis. Chest 2008;133:1261-6.

8. Lu MS, Liu HP, Yeh CH, et al. The role of surgery in hemoptysis causes by thoracic actinomycosis; a forgotten disease. Eur J Cardiothorac Surg 2003;24:694-8.

9. Conant EF, Wechsler RJ. Actinomycosis and nocardiosis of the lung. J Thorac Imaging 1992;7:75-84.

10. Weese WC, Smith IM. A study of 57 cases of actinomycosis over a 36 year period. Arch Intern Med 1975;135:1562-8.

11. McNeil MM, Schaal KP (Actinomyces species [Actinomycoses]). In: Yu VL, Weber R, Raoult D, eds. Antimicrobial Therapy and Vaccines. Volume I: Microbes, 2nd edn. New York: Apple Trees Production <http://www.antimicrobe.org/b73.asp> (Accessed on December 21, 2010).

12. de Fatima Goncalves Ferreira D, Amado J, Neves S, et al. Treatment of pulmonary actinomycosis with levofloxacin. J Bras Pneumol 2008;34:245-8. 


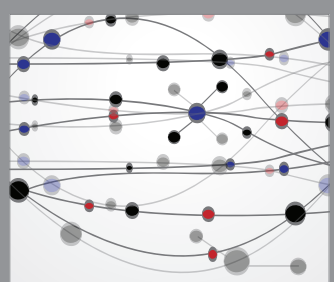

The Scientific World Journal
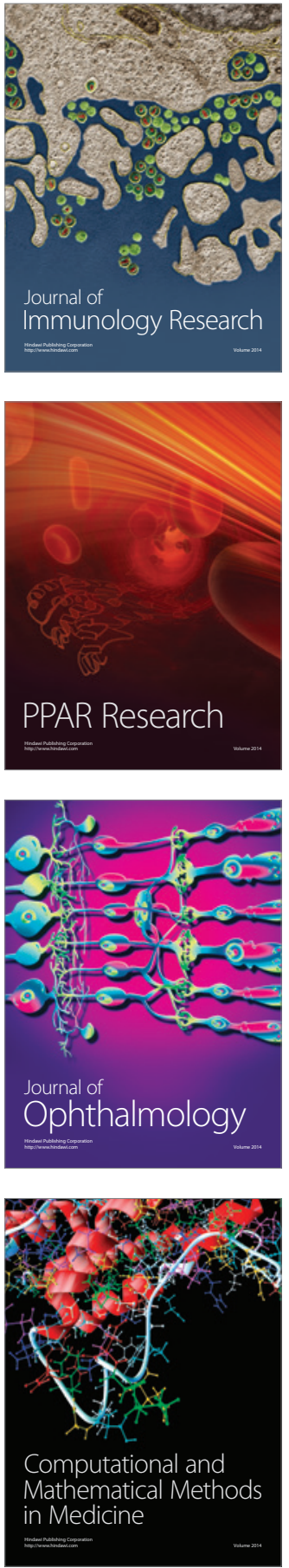

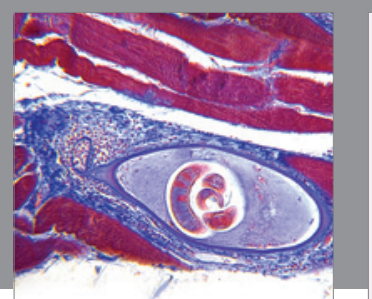

Gastroenterology Research and Practice

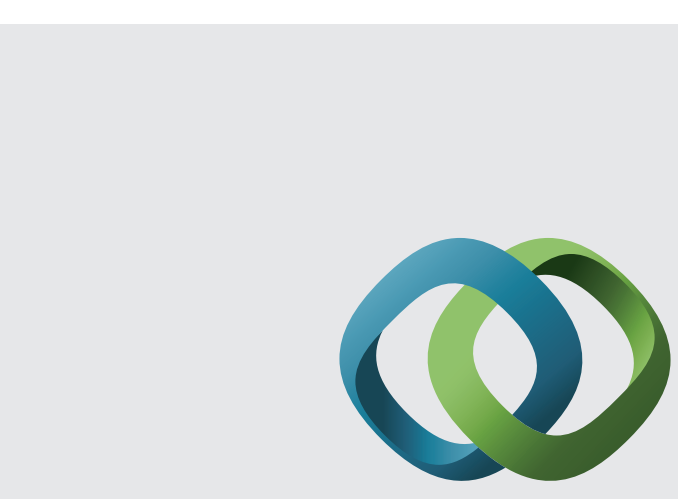

\section{Hindawi}

Submit your manuscripts at

http://www.hindawi.com
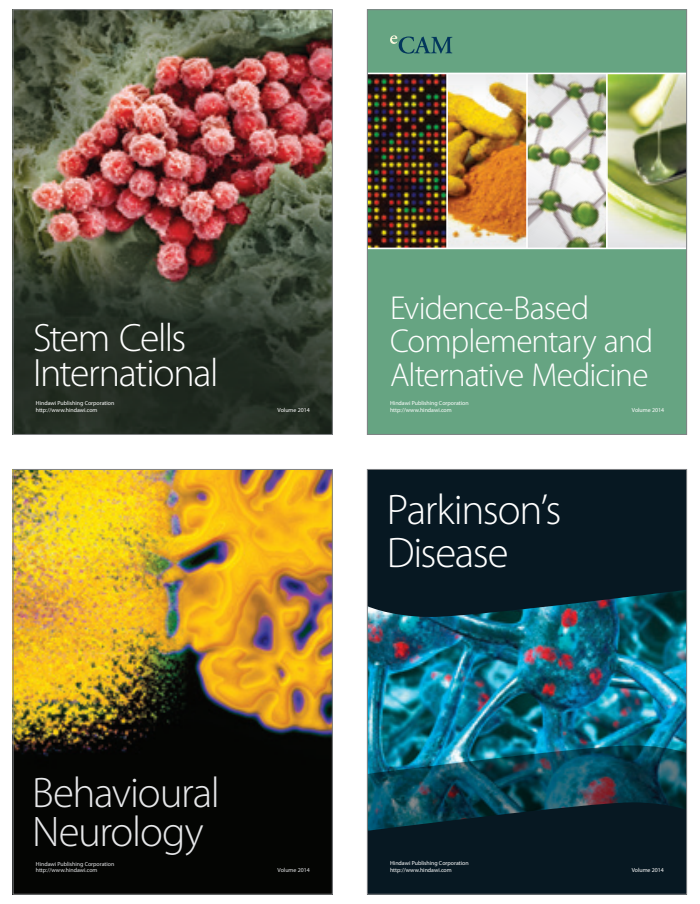
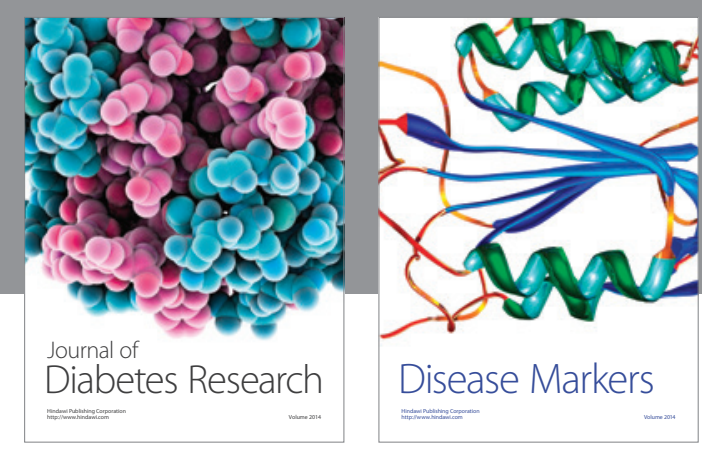

Disease Markers
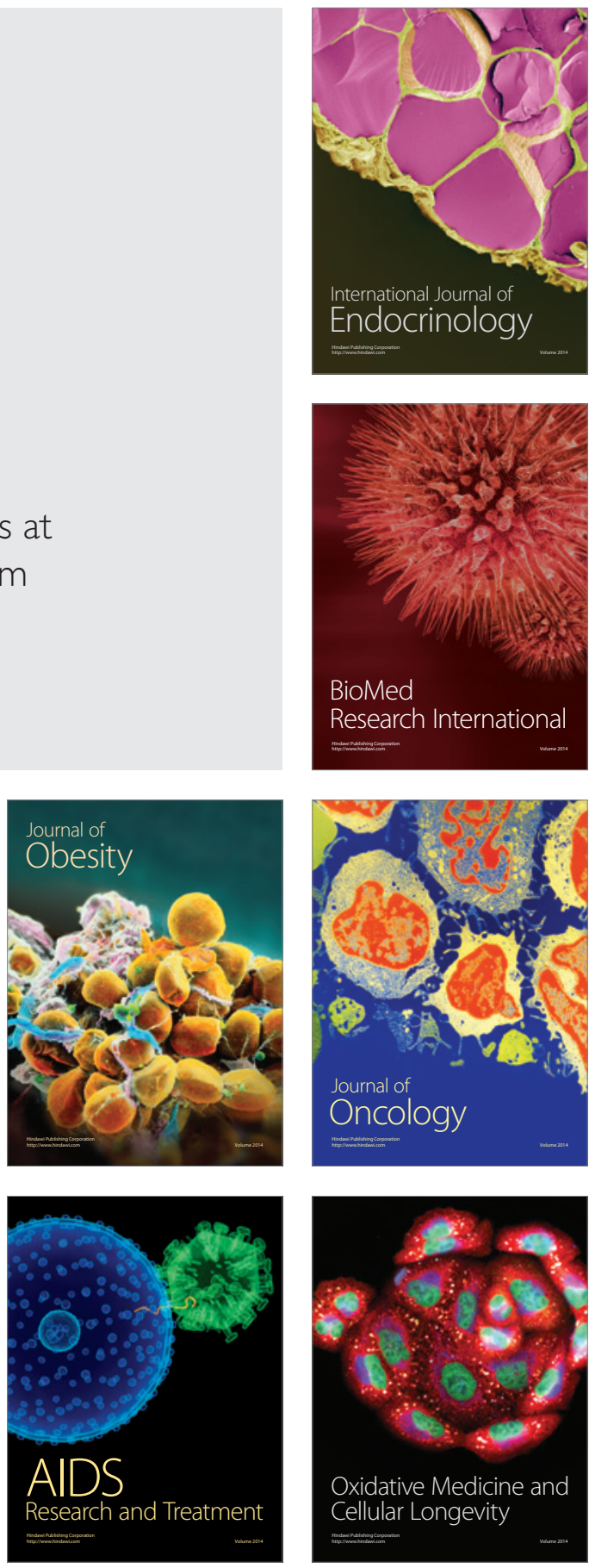